\title{
A Novel Mechanism of Lactobacilli Bacteria Action on Development of Hepatocytic Tolerance to Staphylococcus aureus
}

\author{
Yi-Heng Chiu ${ }^{1}$, Shiao-Lin Lin $^{2}, \&$ Meei-Yn Lin ${ }^{1}$ \\ ${ }^{1}$ Department of Food Science and Biotechnology, National Chung Hsing University, Taichung 40227, Taiwan, \\ ROC \\ ${ }^{2}$ Department of Neurology, Chong Guang Hospital, MiaoLi County35159, Taiwan, ROC \\ Correspondence: Meei-Yn Lin, Department of Food Science and Biotechnology, National Chung Hsing University, \\ Taichung 40227, Taiwan, ROC. Tel: 886-4-2284-0385 ext. 2208. E-mail: mylin@dragon.nchu.edu.tw
}

Received: June 28, 2013 Accepted: July 24, 2013 Online Published: August 15, 2013

doi:10.5539/jas.v5n9p156 URL: http://dx.doi.org/10.5539/jas.v5n9p156

\begin{abstract}
The fundamental characteristics of mammalian PGLYRPs and NOD2 in the reduction of pathogen-induced hepatocytic inflammation as well as interaction with probiotics have not yet been well studied. The aim of this research was to explore whether or not probiotics exert hepatoprotective effects by means of attenuation of NOD2NF- $\mathrm{KB}$ signal transduction induced by Staphylococcus aureus through regulation of PGLYRP2/3. By ELISA analysis of pro-inflammatory cytokine secretion and RT-qPCR assay of NOD2 and PGLYRP2/3 gene expression upon stimulation of bacteria lysates, we found that PGLYRP2 and NOD2 play important roles in the transduction of inflammatory responses induced by $S$. aureus lysates, while PGLYRP3 induced by Lactobacillus plantarum MYL26 lysates serves as an anti-inflammation mediator, counteracting the effect of PGLYRP2 and NOD2. We proposed that one new mechanism by which probiotics exert hepatoprotective effect is through induction of PGLYRP3, which antagonizes PGLYRP2 and NOD2, thus leading to attenuation of NOD2-NFkB signal transduction.
\end{abstract}

Keywords: NOD2, PGLYRP2, PGLYRP3, inflammation, lactic acid bacteria

\section{Abbreviations}

GI tract, gastrointestinal tract; LPS, lipopolysaccharides; PRRs, pattern recognition receptors; TLRs, toll-like receptors; NODs, nucleotide-binding oligomerization domain-containing proteins; PGLYRPs, peptidoglycan recognition proteins; PGNs, peptidoglycans; LTA, lipoteichoic acid

\section{Introduction}

Gastrointestinal tract microbiota and bacterial translocation play imperative characters in the pathogenesis of systemic inflammation, especially liver diseases (Norman et al., 2008). Over proliferation of intestinal bacteria, elevated levels of bacterial lipopolysaccharides, and even worse, increased bacterial translocation from the GI tract give rise to humans more susceptible to severe inflammatory syndrome, thus suffering from a series of systemic disorders (Seo \& Shah, 2012). Impaired GI tract epithelial integrity has been demonstrated as major cause of leaky gut that allowed translocation of bacteria into blood circulation. Bacteria such as Staphylococcus aureus produce numerous toxins triggering inflammatory responses that cause not only foodborne illness but also enhanced gut permeability which predispose to bacterial infections as well as bacterial translocation into bloodstream (Wang et al., 2001). As a result, in addition to improvement of leaky gut, how to develop cellular tolerance to invading pathogens or elevated inflammatory stimuli has became an important issue.

Probiotics have been defined as live microorganisms, when administered in adequate amounts, confer health advantages on the host beyond their intrinsically elementary nutrition (Arora et al., 2013). The most prevalent probiotics used in fermented foods and dietary supplements are from two lactic acid bacteria genera: Bifidobacterium and Lactobacillus (Krznaric et al., 2012).

It is well acknowledged that inherited and acquired factors lead to alterations in GI tract microbiota which might leave hosts susceptible to a wide range of disorders. Recent studies have suggested that infectious diarrhea (Quigley, 2012), various allergies (Noval Rivas et al., 2013), inflammatory bowel diseases (IBDs) and endotoxemia (Caradonna et al., 2000) are related to variations in the GI tract microflora. Variations in the GI 
tract microflora influence susceptibilities not only to intestinal disorders, but also, at least in part, to systemic immune diseases, such as obesity (Aggarwal et al., 2013), cardiovascular diseases (Manco et al., 2010) and diabetes mellitus (Larsen et al., 2010). These diseases are also associated with short chain fatty acid produced by GI tract microorganisms due to its regulation capacities on appetite hormones (Conterno et al., 2011). Therefore, any intrinsic or extrinsic factors affecting microbial homeostasis could have a substantial impact on human health.

The innate immune system detects pathogenic bacteria by means of a collection of pattern recognition receptors highly conserved from insects to humans and shows specificity for microorganism cellular components not found in eukaryotes (Brenner et al., 2013). Pathogen-associated molecular patterns (PAMPs), which are molecules or structures that exist on a class of pathogens, are recognized by PRRs, such as toll-like receptors, nucleotide-binding oligomerization domain-containing proteins, and peptidoglycan recognition proteins (Inoue \& Shinohara, 2013). The most characteristic bacterial PAMPs consist of LPS derived from Gram-negative bacteria and peptidoglycans originating from either Gram-positive or Gram-negative bacteria. In addition to cell wall components, both viral/ bacterial unmethylated CpG motifs and flagellin are also powerful PAMPs (Fujita \& Taguchi, 2012). In addition to genus of mycoplasma which lacks cell wall structures, PGN is a ubiquitous component of bacteria cell walls, and is an extraordinarily distinguishable antigen for characterizing pathogenic bacteria. Over the past few decades, several lines of evidence have indicated that PGNs are recognized by at least three PRRs, including NOD2, PGLYRPs, and TLR2.

NOD2, also referred to as caspase recruitment domain-containing protein 15, is a member of the NOD1/Apaf-1 family (Balamayooran et al., 2010), which recognizes the bacterial cell wall PGN that contains the specific structure muramyl dipeptide. It has been shown that NOD2 are highly relevance to bacteria-induced inflammation (Franchi et al., 2008).

Mammalian PGLYRPs can be categorized into four types: PGLYRP1, PGLYRP2, PGLYRP3 and PGLYRP4 (Sorbara \& Philpott, 2011). PGLYRP1, PGLYRP3, and PGLYRP4 have been proposed to have bactericidal properties and are primarily expressed in polymorphonuclear leukocytes. PGLYRP2, an $\mathrm{N}$-acetylmuramoyl-L-alanine amidase, is mainly expressed in the liver and sequentially released into the systemic circulation. Mammalian PGLYRPs were originally considered PRRs. However, they not only function as PRRs, but also participate in pro- and anti-inflammatory responses (Boneca, 2009).

NOD2 tolerance is most likely a consequence of the host's defence system which confines pro-inflammatory actions when stimulation of innate immunity by PGN originates from both Gram-positive and Gram-negative bacteria (Macho et al., 2011). A growing body of research has indicated that contact of lymphocytes or intestinal epithelial cells with either Gram-negative or Gram-positive bacteria cell wall constituents results in refractoriness to subsequent challenge (Faria et al., 2012). This is important for the development of a potential treatment for inflammatory disorders. In this context, a number of reports have demonstrated that NOD2 tolerance does not only occur due to PGN, but also to many other bacteria cellular constituents, such as glycolipids, LPS, lipoteichoic acid, flagellin, unmethylated CpG DNA motif, as well as several heat shock proteins (Kim et al., 2011; Muller-Anstett et al., 2010).

To our knowledge, there are only a limited number of comprehensive studies that have addressed the subject of probiotic-induced NOD2 tolerance in liver cells. As a result, we intensified our efforts to investigate the mechanistic actions by which probiotics exert hepatoprotective effects via development of NOD2 tolerance.

In this study, seven strains of probiotics (L. plantarum MYL26, L. plantarum MYL31, L. acidophilus MYL201, L. acidophilus MYL202, L. bulgaricus MYL101, L. bulgaricus MYL102 and L. casei MYL01) and pathogenic bacteria $S$. aureus BCRC 10451 were processed independently into heat-killed bacteria lysates, which were used as stimuli to induce pro- and anti-inflammatory processes. Enzyme-linked immunosorbent assay (ELISA) was performed to evaluate and quantify the inflammation-associated protein expression of tumour necrosis factor alpha (TNFa), interleukin 6 (IL-6), interleukin 8 (IL8), interleukin 12 (IL-12), NF-kB nuclear p65 and

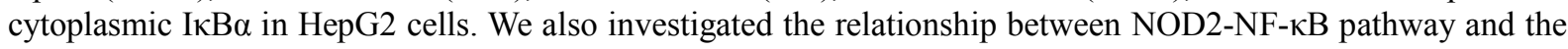
expressions of PGLYRP2/3 upon stimulation of probiotics and $S$. aureus lysates. Moreover, siRNA technique was conducted on NOD2 and PGLYRP2/3 to observe whether NOD2 and PGLYRP2/3 play essential roles in the induction of inflammation tolerance which might lead to a promising therapy for liver injury induced by $S$. aureus. 


\section{Material and Methods}

\subsection{Bacterial Strains}

Isolation and identification of lactic acid bacteria from newborn infant faeces and breast milk were performed in the Microbiology Laboratory of the Department of Food Science and Biotechnology, National Chung Hsing University, Taichung, Taiwan. Staphylococcus aureus BCRC 10451 was acquired from the Bioresource Collection and Research Centre (BCRC, Hsinchu, Taiwan). Lactic acid bacteria were grown anaerobically at $37^{\circ} \mathrm{C}$ in MRS (Difco Laboratories, Detroit, MI, USA). S. aureus was grown aerobically at $37^{\circ} \mathrm{C}$ in terrific broth (1.2\% peptone, $2.4 \%$ yeast extract, $72 \mathrm{mM} \mathrm{K}_{2} \mathrm{HPO}_{4}, 17 \mathrm{mM} \mathrm{KH}_{2} \mathrm{PO}_{4}$, and $0.4 \%$ glycerol). All strains were serially subcultured three times prior to use.

\subsection{Preparation of Heat-Killed Bacteria Lysates}

Bacteria were stored at $-80^{\circ} \mathrm{C}$ until use. All bacterial strains were cultured at $37^{\circ} \mathrm{C}$ for $16 \mathrm{~h}$ and collected by centrifugation at $2500 \mathrm{rpm}$ for $5 \mathrm{~min}$. For preparation of bacteria lysates, cells were adjusted to $1 \times 10^{8} \mathrm{cfu} / \mathrm{mL}$ and then washed twice with deionized water before being suspended in phosphate-buffered saline (PBS). Repeated freezing and thawing method was adopted for preliminary cell disruption. Then, cells were heat-killed at $75^{\circ} \mathrm{C}$ for $3 \mathrm{~min}$, followed by homogenization by Tissue-Tearor (Biospec, USA). All bacteria lysates were aliquoted $10 \mu \mathrm{L}$ for accessing whether or not there were existing live bacteria.

\subsection{Cell Culture}

Human hepatocellular carcinoma cells (HepG2) were obtained from the Bioresource Collection and Research Centre (BCRC, Hsinchu, Taiwan). The cells were cultured in Dulbecco's modified Eagle's medium (DMEM) supplemented with $10 \%$ heat-inactivated foetal bovine serum (FBS) at $37^{\circ} \mathrm{C}$ in a humidified $(95 \%)$ atmosphere with $5 \% \mathrm{CO}_{2}$.

\subsection{Stimulation of HepG2 Cells with Probiotics Followed by S. aureus Challenge}

HepG2 cells $\left(1 \times 10^{6}\right.$ cells $\left./ \mathrm{mL}\right)$ were treated with probiotic lysates at $37^{\circ} \mathrm{C}$ for 20 hours. After stimulation, HepG2 cells were challenged with $S$. aureus lysates for 20 hours. The supernatants were harvested and assayed IL-6, IL-8, IL-12 and TNF- $\alpha$. The adhesion cells were lysed for detection of nuclear NF- $\mathrm{kB}$ p 65 and cytoplasmic I $\mathrm{B} \alpha$. Nuclear/ cytoplasmic proteins were collected using Nuclear/Cytosol Fractionation Kit (Biovision, USA), and samples were suspended with buffer containing protease inhibitor cocktail. All the protein expressions were evaluated and quantified by ELISA according to manufacturers' instruction (eBioscience ELISA system). Housekeeping protein human actin was adopted as internal control.

\subsection{MTT Assay}

3-[4,5-dimethyl-2-thiazolyl]-2,5-diphenyl-2H-tetrazolium bromide (MTT) assay is based on the cleavage of the tetrazolium salt by mitochondrial dehydrogenases in viable cells. In order to determine the most appropriate co-incubation times, approximately $1 \times 10^{5}$ cells were plated onto each well of 96 -well plates for $24 \mathrm{~h}$, followed by treatment with $S$. aureus and different probiotic lysates for 5, 10, 15, 20, 25 and 30 hours. After incubation, $200 \mathrm{~mL}$ of MTT solution $(0.5 \mathrm{mg} / \mathrm{mL})$ were added to each well for $4 \mathrm{~h}$. The supernatant was removed and 200 $\mu \mathrm{L}$ of dimethyl sulphoxide (DMSO) were added to each well to dissolve the dark blue formazan crystals. The absorbance was measured at $570 \mathrm{~nm}$.

\subsection{RT- $q P C R$}

RNA isolation was conducted using phenol-chloroform method. Comparisons of gene expressions via qPCR were performed by designing the following primer: GAPDH (5'- GAG TCA ACG GAT TTG GTC GT-3' and 5'- TTG ATT TTG GAG GGA TCT CG -3'), PGLYRP2 (5'- ATG GCT CTC CAG ATG TCA CC-3' and 5'GGA AGG CCA TGG TTA ACA GA -3'), PGLYRP3 (5'- TCA CAT GGA CAC ACG GAA CT -3' and 5'GGA TGT TGA CCA CGT CAC TG -3'), and NOD2 (5'- CTG TCC AGA CCC TGC TCT TC -3' and 5'- TTG GAC ACC ATC CAT GAG AA -3'). Quantitative PCR was carried out by the condition: 2 min at $55^{\circ} \mathrm{C}, 10 \mathrm{~min}$ at $94^{\circ} \mathrm{C}, 40$ cycles of $15 \mathrm{~s}$ at $94^{\circ} \mathrm{C}$, and $1 \mathrm{~min}$ at $60^{\circ} \mathrm{C}$. Human $G A P D H$ mRNA is adopted as internal control.

\subsection{Statistical Analysis}

All values are expressed as mean \pm standard deviation (SD). Each value is the mean of three separate experiments in each group $(\mathrm{n}=3$ ). Statistical comparisons were carried out by student's t test. $p<0.05$ (symbol *) was considered significantly different. 


\section{Results}

3.1 Lactobacilli Bacteria Treatment Attenuated S. aureus-Induced Pro-Inflammatory Cytokine Secretions

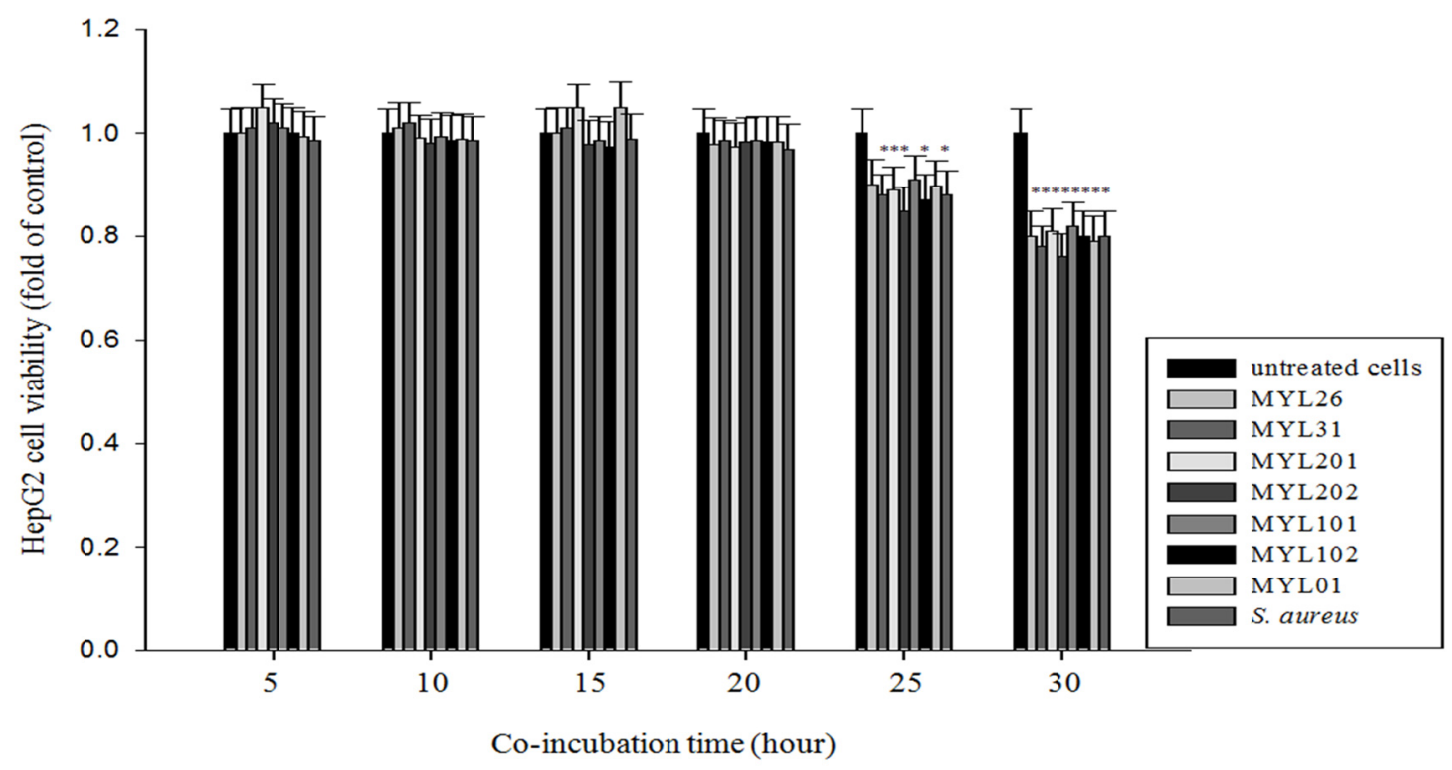

Figure 1 . HepG2 cell viability was assayed by MTT method. The relative cell viability was expressed as the mean fold of those of untreated cells. Symbol * represents $p<0.05$ assayed by student's $t$ test $(n=3)$

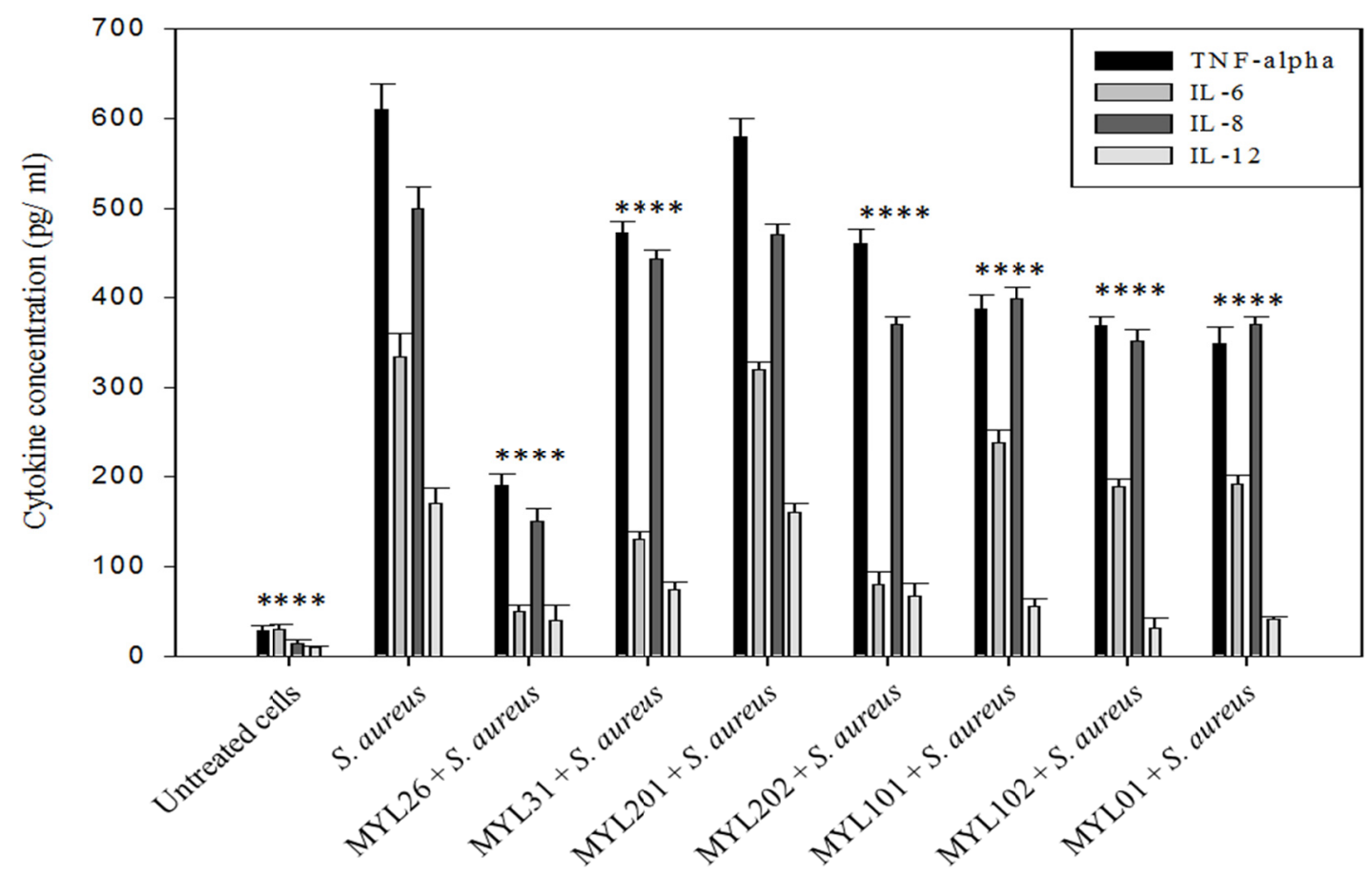

Figure 2. Cytokine secretions by stimulation of HepG2 cells with lactobacilli bacteria lysates for 20 hours followed by challenge of $S$. aureus lysates for 20 hours. Each cytokine secretion between groups was compared with that of $S$. aureus challenge group assayed by student's t test. Symbol * represents $p<0.05(\mathrm{n}=3)$ 
Before co-incubation of HepG2 cells and bacteria lysates, we perform MTT assay to assess the most appropriate incubation time. The data of Figure 1 indicated that HepG2 cell viability was not influenced within 20 hours. To address the development of HepG2 cell tolerance to inflammation, HepG2 cells were exposed to lactobacilli bacteria lysates, followed by challenge of $S$. aureus lysate. In Figure 2, each cytokine secretion between groups was compared with that of $S$. aureus challenge group assayed by student's t test. As Figure 2 showed, the production of TNF- $\alpha$, IL-6, IL-8, and IL-12 decreased to a greater extent than that of $S$. aureus challenge group. Among these seven lactobacilli bacteria, $L$. plantarum MYL26 exerted the most efficient anti-inflammatory effect, while $L$. acidophilus MYL201 did not reduce inflammation significantly compared with that of $S$. aureus challenge group. As a consequence, we adopted L. plantarum MYL26 and L. acidophilus MYL201 as stimuli in the following research to investigate the molecular mechanism by which lactobacilli bacteria elicit their anti-inflammatory function.

3.2 Lactobacillus Plantarum MYL26 Treatment Significantly Induced the Expressions of PGLYRP3 and the Cytoplasmic I $\mathrm{K} B \alpha$

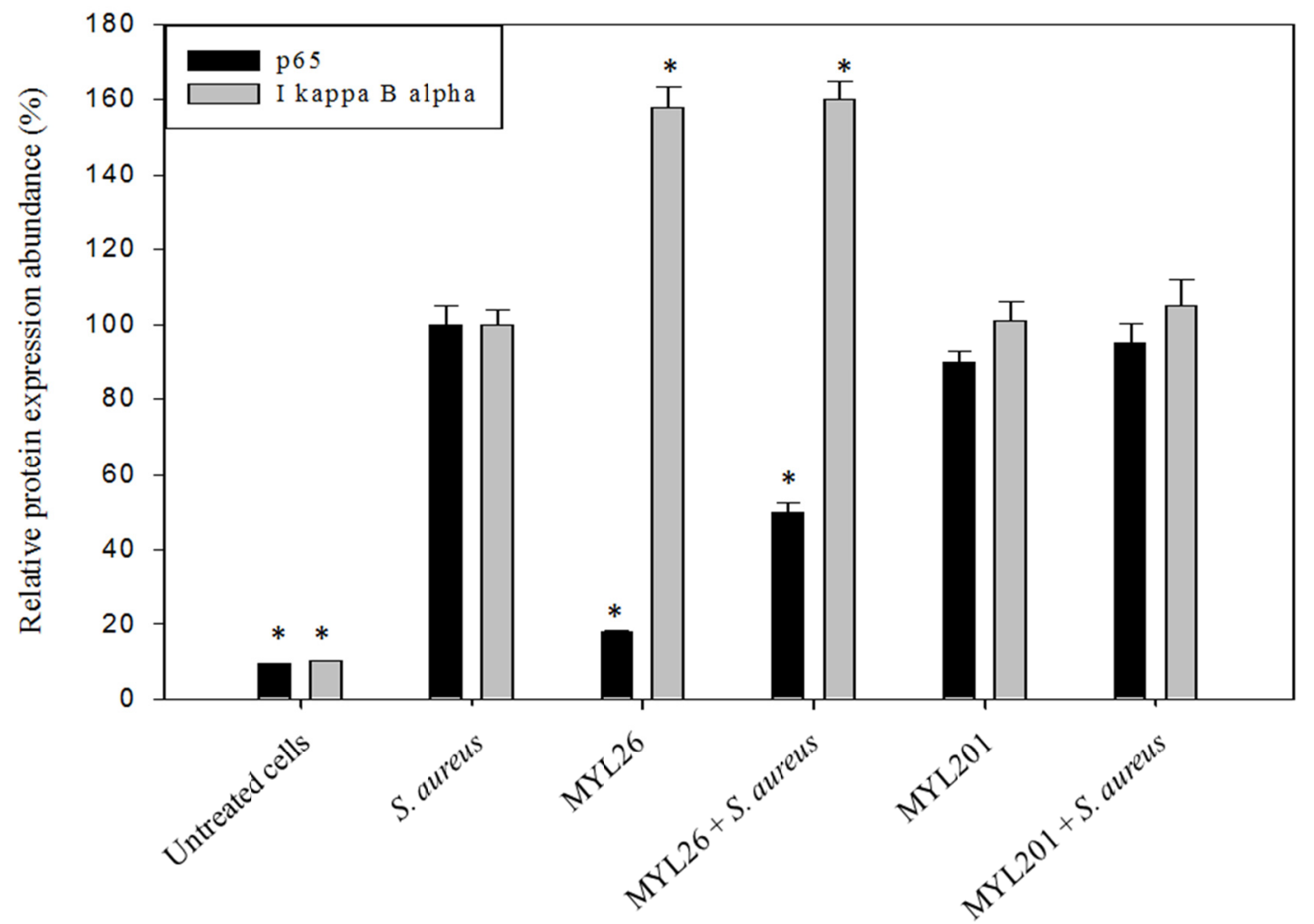

Figure 3. Bacteria lysates stimulation affected nuclear translocation of NF- $\mathrm{kb}$. The percentage of protein abundance in each group was normalized to that of $S$. aureus challenge group $(100 \%)$. Relative protein expression between groups was compared with that of $S$. aureus challenge group assayed by student's $t$ test. Symbol * represents $p$ $<0.05(\mathrm{n}=3)$

S. sureus: S. sureus lysates challenge for 20 hours;

MYL26/ MYL201: lactobacilli bacteria lysates stimulation for 20 hours;

MYL26/ MYL201+ S. sureus: prior stimulation of lactobacilli bacteria lysates for 20 hours followed by challenge of $S$. sureus lysates for 20 hours.

In addition to assessment of pro-inflammatory cytokines, ELISA was performed to quantify the nuclear translocation of p65 and cytoplasmic expression of IкB $\alpha$. In figure 3/4, the percentage of protein (or cDNA) abundance in each group was normalized to that of S. aureus challenge group (100\%). Relative protein (or cDNA) abundance between groups was compared with that of $S$. aureus challenge group assayed by student's test. As figure 3 showed, exposure to L. acidophilus MYL201 lysates induced p65 nuclear translocation similar to that of $S$. aureus challenge group, while exposure to L. plantarum MYL26 lysates only slightly induced p65 translocation 
but dramatically induced cytoplasmic expression of IкB $\alpha$. This result was in line with the data of Figure 1 which showed L. plantarum MYL26 had potent anti-inflammation capacity while L. acidophilus MYL201 did not.

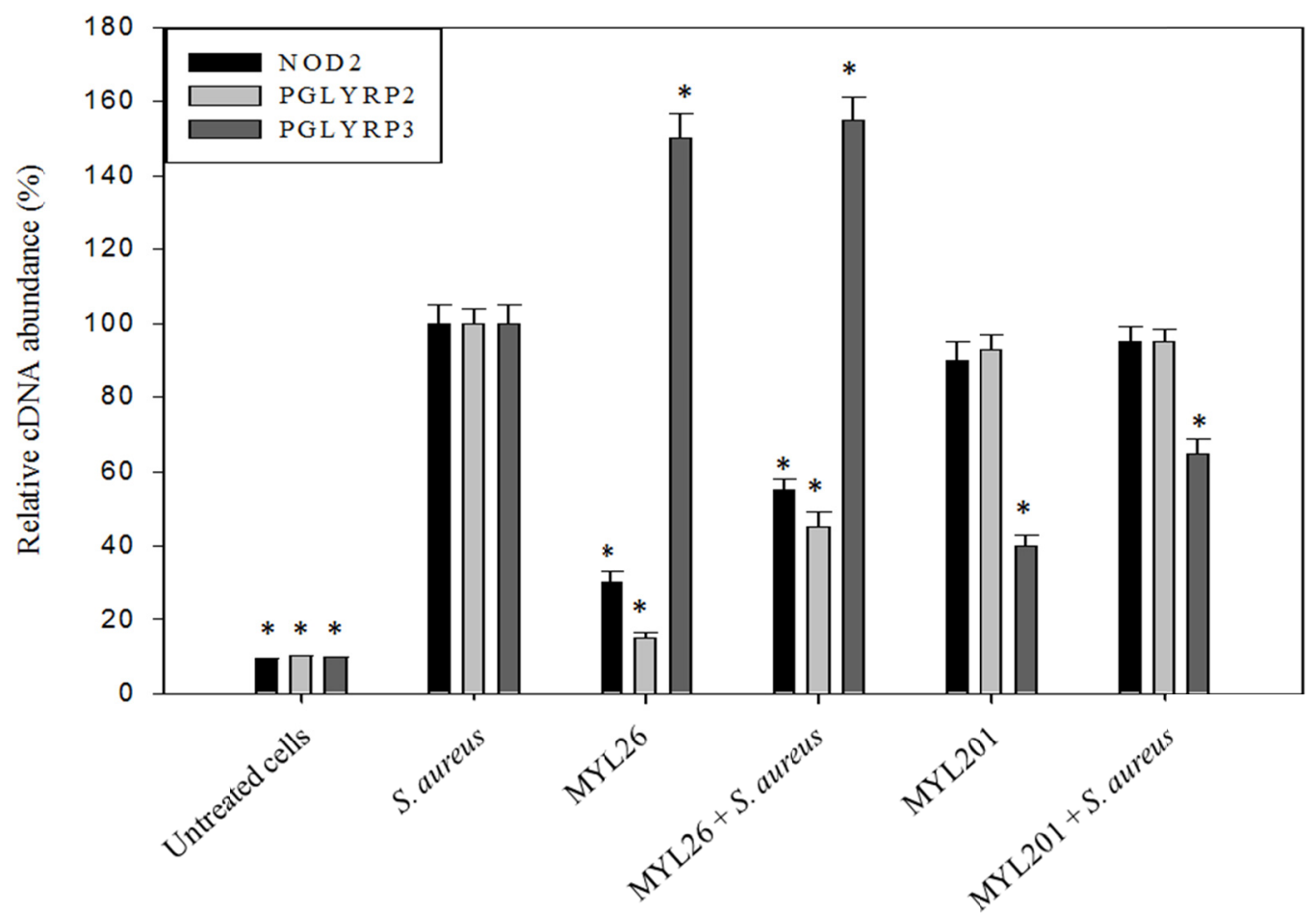

Figure 4. Bacteria lysates stimulation affected gene expressions of NOD2 and PGLYRP2/3. The percentage of cDNA abundance in each group was normalized to that of $S$. aureus challenge group (100\%). Relative cDNA abundance between groups was compared with that of $S$. aureus challenge group assayed by student's t test. Symbol * represents $p<0.05(\mathrm{n}=3)$

S. sureus: S. sureus lysates challenge for 20 hours;

MYL26/ MYL201: lactobacilli bacteria lysates stimulation for 20 hours;

MYL26/ MYL201+ S. sureus: prior stimulation of lactobacilli bacteria lysates for 20 hours followed by challenge of $S$. sureus lysates for 20 hours.

To further investigate the molecular mechanisms responsible for the development of tolerance to $S$. aureus in HepG2 cells when prior treated with lactobacilli bacteria lysates, we conducted RT-qPCR to detect the NOD2 and PGLYRP2/3 gene expressions in HepG2 cells upon stimulation of $S$. aureus lysates alone, lactobacilli bacteria lysates alone as well as prior stimulation of lactobacilli bacteria followed by challenge of $S$. aureus. Figure 4 showed that $L$. plantarum MYL26 treatment alone or challenge of $S$. aureus after treatment with $L$. plantarum MYL26, significantly induced PGLYRP3 expression, while L. acidophilus MYL201, which was prior shown unable to reduce inflammation, did not induce PGLYRP3 but induce PGLYRP2 and NOD2 as great degrees as that of $S$. aureus challenge group.

One interesting finding worth noting was that increased pro-inflammatory cytokine secretions (Figure2) as well as nuclear p65 (figure 3) were in accordance with enhanced expressions of NOD2 and PGLYRP2 (Figure 4), but had inverse correlation with enhanced expression of PGLYRP3.

\subsection{NOD2 and PGLYRP2/3 Knockdown Affected Pro-Inflammatory Cytokine Profile}

Since we found that NOD2 and PGLYRP2/3 expressions are relevance to pro-inflammatory cytokine secretion and NF- $\kappa$ B activity, we conducted siRNA technique for knockdown of NOD2 and PGLYRP2/3 expression in the aim of exploring to what extent NOD2 and PGLYRP2/3 influences inflammatory process. In Figure 5, each cytokine secretion was compared with that of wild type HepG2 cells by student's $t$ test. In Figure 6/7/8, the 
percentage of cDNA abundance in each group was normalized to that of $S$. aureus challenge group of wild type $(100 \%)$. Relative cDNA abundance between groups was compared with that of wild type assayed by student's $t$ test.

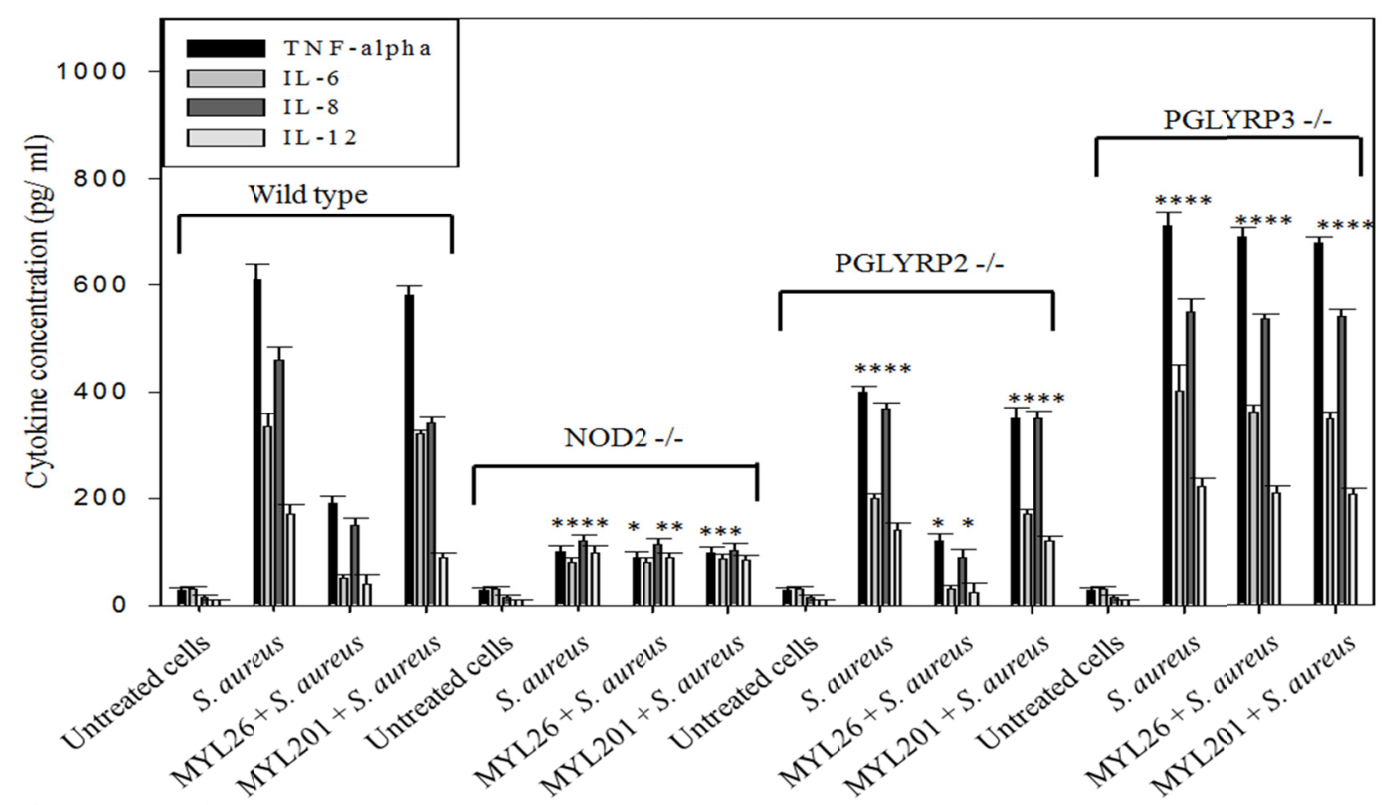

Figure 5. NOD2 and PGLYRP2/3-silenced HepG2 cells were treated with lactobacilli bacteria lysates for 20 hours, followed by challenge of $S$. sureus lysates for 20 hours. Each cytokine secretion between groups was compared with that of wild type assayed by student's $\mathrm{t}$ test. Symbol * represents $p<0.05(\mathrm{n}=3)$

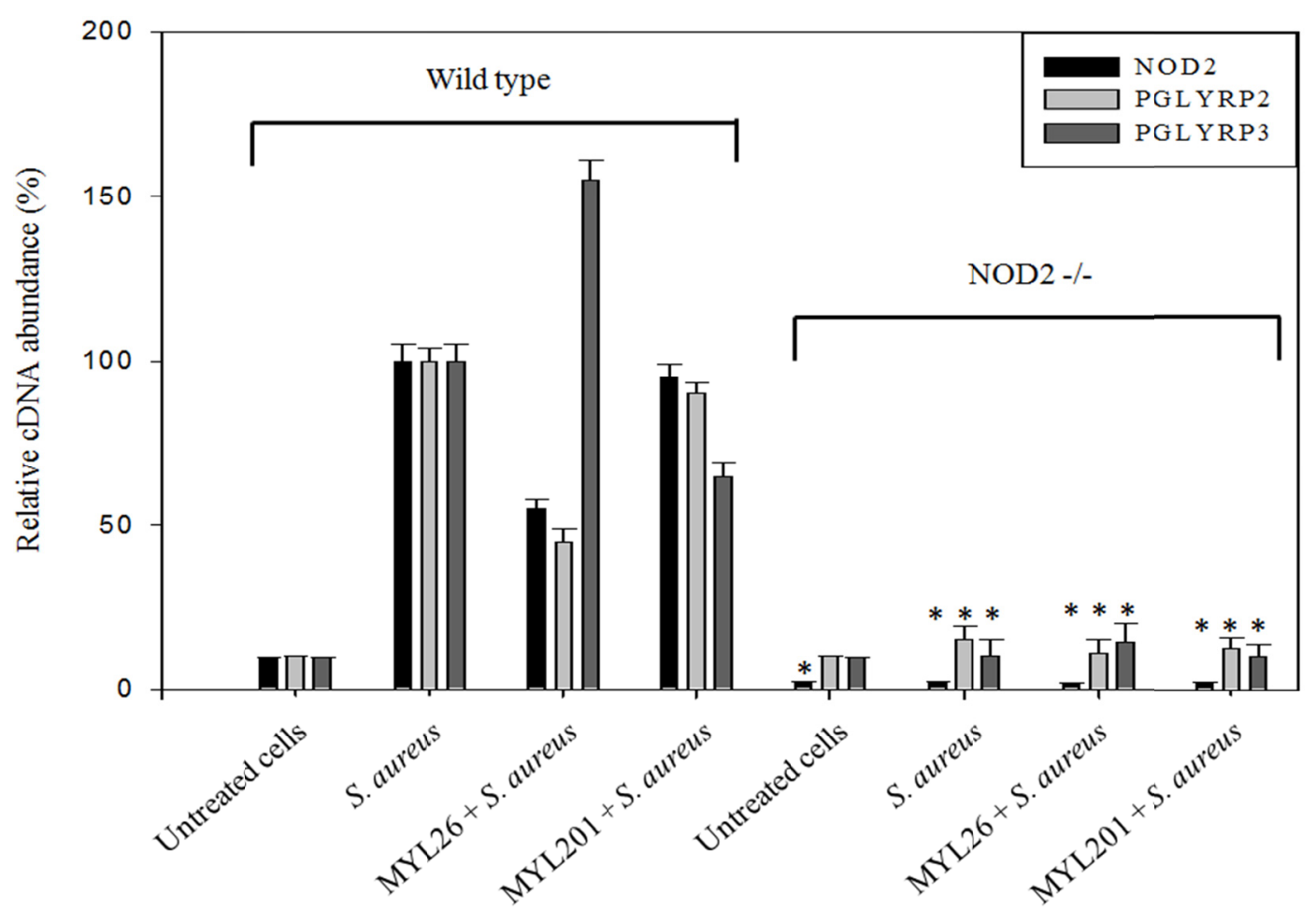

Figure 6. Bacteria lysates stimulation affected NOD2-silenced HepG2 cell gene expressions of PGLYRP2/3. The percentage of cDNA abundance in each group was normalized to that of $S$. aureus challenge group of wild type $(100 \%)$. Relative cDNA abundance between groups was compared with that of wild type assayed by student's $t$ test. Symbol * represents $p<0.05(\mathrm{n}=3)$ 


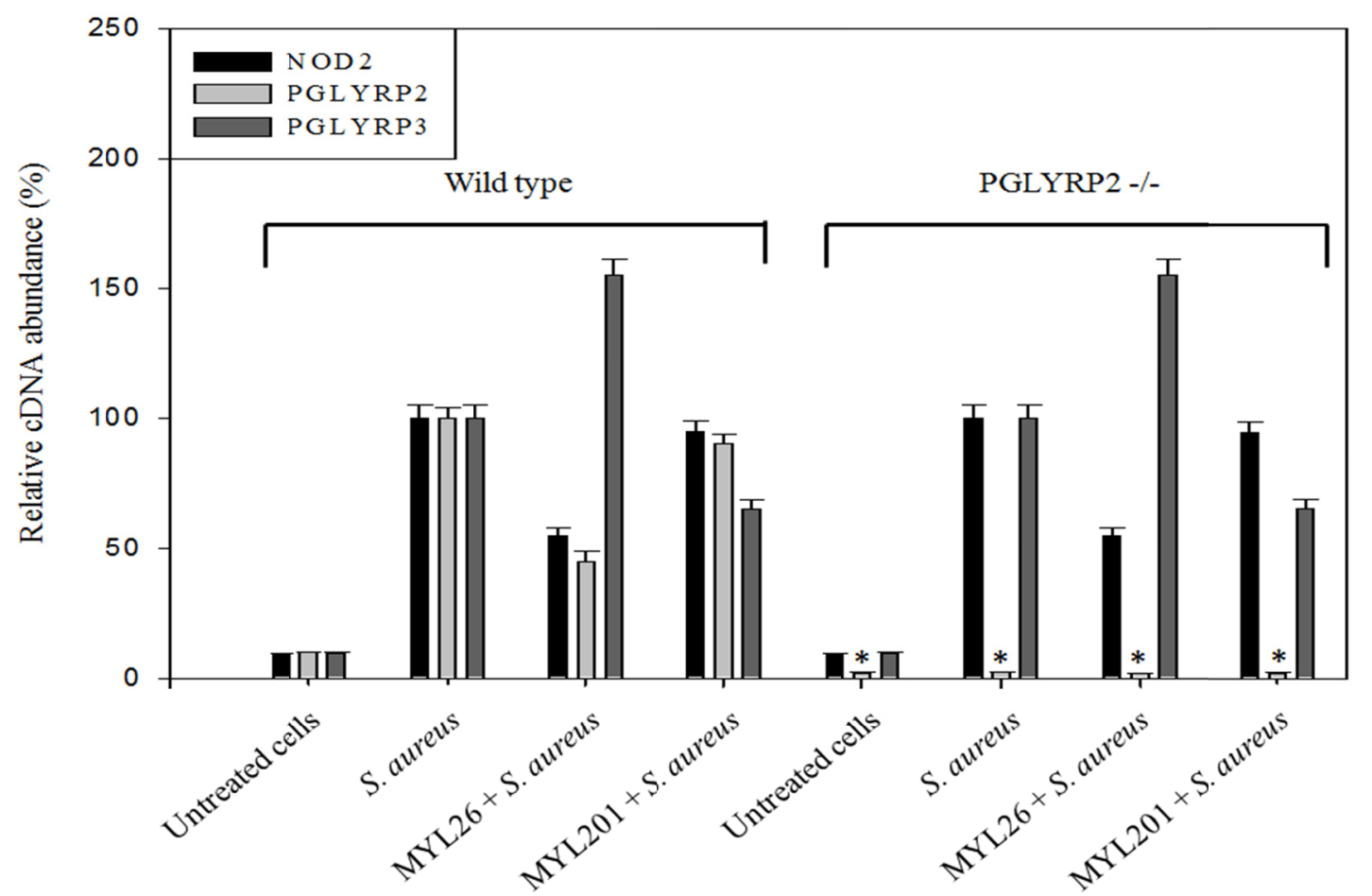

Figure 7. Bacteria lysates stimulation affected PGLYRP2-silenced HepG2 cell gene expressions of NOD2 and PGLYRP3. The percentage of cDNA abundance in each group was normalized to that of $S$. aureus challenge group of wild type (100\%). Relative cDNA abundance between groups was compared with that of wild type assayed by student's $\mathrm{t}$ test. Symbol * represents $p<0.05(\mathrm{n}=3)$

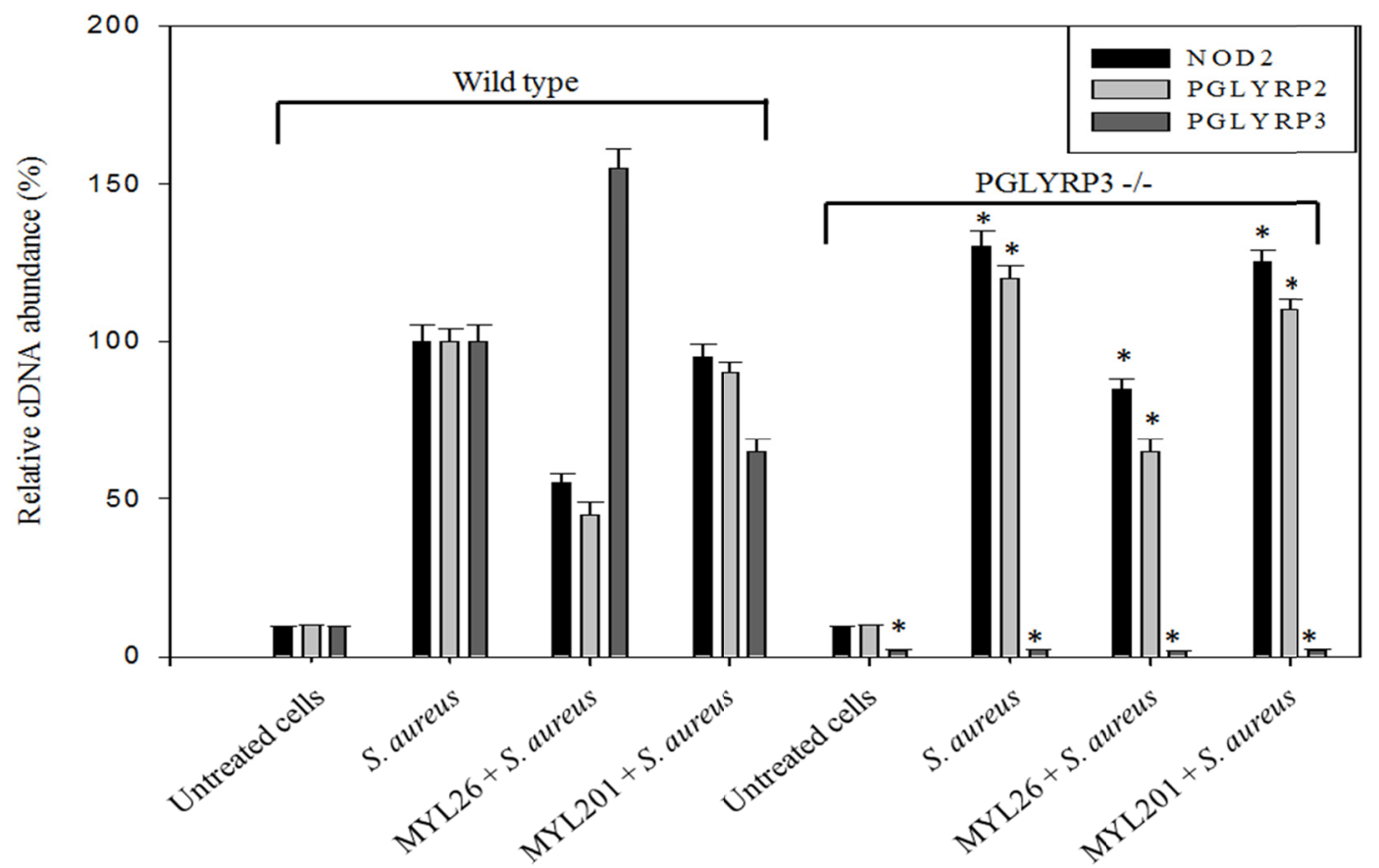

Figure 8. Bacteria lysates stimulation affected PGLYRP3-silenced HepG2 cell gene expressions of NOD2 and PGLYRP2. The percentage of cDNA abundance in each group was normalized to that of $S$. aureus challenge group of wild type (100\%). Relative cDNA abundance between groups was compared with that of wild type assayed by student's $\mathrm{t}$ test. Symbol * represents $p<0.05(\mathrm{n}=3)$ 
Figure 5 showed that NOD2 knockdown had an enormous impact on the pro-inflammatory properties of $S$. aureus. NOD2 inactivation not only led to abolishment of pro-inflammatory cytokine secretion induced by $S$. aureus, but also gave rise to abolishment of PGLYRP2/3 expression as Figure 6 showed. The expressions of PGLYRP2/3 induced by $S$. aureus, L. acidophilus MYL201 and L. plantarum MYL26 lysates were observed dramatically decreased when NOD2 was silenced, while PGLYRP2 knockdown (Figure 7) did not affect the expression of NOD2 and PGLYRP3. Figure 5 also revealed that the effect of PGLYRP2 knockdown, even though significant, was not as potent as that of NOD2 knockdown on the inactivation of $S$. aureus-induced inflammatory process, as evidenced by pro-inflammatory cytokine secretion. The observation that PGLYRP2 knockdown made S. aureus partially losses pro-inflammatory properties suggested that PGLYRP2 might mediate inflammation. As for PGLYRP3 knockdown, it was showed that PGLYRP3 knockdown significantly affected both the anti-inflammatory functions of lactobacilli bacteria and the inflammatory properties exerted by $S$. aureus. PGLYRP3-silenced HepG2 cells challenged by S. aureus lysates secreted more pro-inflammatory cytokines than that of wild type. Moreover, L. plantarum MYL26 did not exert anti-inflammatory capacity when PGLYRP3 was silenced. Although PGLYRP2 knockdown did not affect NOD2 and PGLYRP3 expression, the result of figure 8 revealed that PGLYRP3 knockdown considerably increased the expression of NOD2 and PGLYRP2, both of them prior shown to be relevance to pro-inflammation process.

Taken together, the cytokine secretion consequence of NOD2 and PGLYRP2/3 knockdown implicated that NOD2 plays an imperative character in the onset of $S$. aureus-induce inflammation as well as a key mediator of PGLYRP2/3 activation. PGLYRP2 also plays a role in inflammation mediation but the effect is not as profound as NOD2. PGLYRP3 is of importance in limitation of NOD2 and PGLYRP2 expression, the function that might contribute to anti-inflammation.

\section{Discussion}

In view of the fact that NOD2 is associated with enhanced pro-inflammatory cytokine secretion and nitric oxide generation (Cartwright et al., 2007; Scott et al., 2010; Stoffels et al., 2004), we hypothesized that the hepatoprotective effects of probiotics are in part mediated by inhibition of NOD2- NF-kB signal transduction. The aim of this research was to explore whether or not probiotics exert hepatoprotective effects by means of attenuation of NOD2- NF- $\mathrm{kB}$ signal transduction through regulation of PGLYRP2/3.

Within the in vitro study of HepG2, we investigated the induction of inflammation tolerance by stimulation of different strains of lactobacilli bacteria in terms of suppressed pro-inflammatory cytokine secretion in response to challenge of $S$. aureus. In a system of $S$. aureus-induced inflammatory liver damage, we first examined to what extent the practical potency of inflammation tolerance is induced by lactobacilli bacteria. In order to shape the mechanism more elaborately and address the differences from molecular aspects between potent and impotent strains, the most effective and ineffective lactobacilli bacteria strains were selected for exploration of inflammation signal transduction events.

The expressions of NOD2 after treatment with $S$. aureus and lactobacilli bacteria lysates were accessed and found that all bacteria lysates were able to activate NOD2 expression, especially $S$. aureus lysates showing the greatest effect. This outcome was in line with literatures focus on innate immunity regulated by lactobacilli bacteria (Foligne et al., 2007; Matuchansky, 2012; Shida et al., 2009). Regarding the marginal effect of NF- $\kappa B$ p65 nuclear translocation and mild pro-inflammatory cytokine production, we verified that NOD2 expression activated by L. plantarum MYL26 did not lead to serious inflammation. There are a number of points worth noting. First, potent strain L. plantarum MYL26 enhanced NOD2 expression, even if they did not give rise to severe inflammation. Second, NOD2 and PGLYRP2/3 activation was strain-dependent. Each probiotic strain contributed to varying degrees of gene activation. Potent strain L. plantarum MYL26 activated PGLYRP3 much more significantly that that of impotent strain L. acidophilus MYL201, while NOD2 and PGLYRP2 expression were showed much higher in group of impotent strain L. acidophilus MYL201. Third, the patterns of NOD2 and PGLYRP2 expression induced by impotent strain L. acidophilus MYL201 lysates were similar to that of $S$. aureus lysates challenge group. These observations implied that NOD2 and PGLYRP2 are relevance to inflammation signal transduction. However, PGLYRP3 seems to play a key role in the development of inflammation tolerance.

To further demonstrate the postulation that PGLYRP2 is relevance to inflammation process and PGLYRP3 contributes to the development of inflammation tolerance, complementary studies are required to detail the relationship between NOD2, PGLYRP2 and PGLYRP3. We conducted siRNA technique targeting PGLYRP2, PGLYRP3 and NOD2, followed by advanced assessment of pro-inflammatory cytokine production. NOD2 knockdown led to significantly decreased pro-inflammatory cytokine production, accompanied by reduced 
expressions of PGLYRP2 and PGLYRP3. One explanation for this is that PGLYRP2 and PGLYRP3 are the downstream signal transducers of NOD2 which is thought to be of great importance in increased secretion of pro-inflammatory cytokine induced by $S$. aureus, as well as lactobacilli bacteria. The result that NOD2 inactivation developed resistance to $S$. aureus corresponded to numerous literatures that indicate $S$. aureus mediates inflammation, in addition to exotoxin, mainly due to cell wall PGN specific to NOD2 (Juarez-Verdayes et al., 2013; Mitchell et al., 2007; Volz et al., 2010).

PGLYRP2 knockdown did not influence the expression of NOD2, the result which provides further evidence that NOD2 serves as an upstream mediator of PGLYRP2. Decreased pro-inflammatory cytokine production caused by PGLYRP2 knockdown, although significant, was not as potent as that caused by NOD2 knockdown. Regarding the phenomenon of increased pro-inflammatory cytokine production and enhanced NOD2 and PGLYRP2 expression, L. plantarum MYL26, at least in part, were deprived of anti-inflammatory efficacies when knockdown of PGLYRP3. Moreover, the observation that potent strain L. plantarum MYL26 was effective in the activation of PGLYRP3 while impotent strain L. acidophilus MYL201 was not, corresponds with the result that PGLYRP3 knockdown abolishes the anti-inflammatory efficacies of $L$. plantarum MYL26.

The results of PGLYRP3 knockdown might be explained in relation to interrupted development of inflammation tolerance. In other words, induction of PGLYRP3 by L. plantarum MYL26 might give rise to anti-inflammatory effect before $S$. aureus challenge. Thus, PGLYRP3 knockdown leads to weak development of inflammation tolerance. However, we also found that PGLYRP3 knockdown led to enhanced expression of NOD2 and PGLYRP2. Combining with the result of poor PGLYRP3 expression caused by NOD2 inactivation, this implies that PGLYRP3 acts as a negative regulator responsible for NOD2 and PGLYRP2 feedback control. It is reasonable to conclude that PGLYRP2 and PGLYRP 3 act as pro- and anti-inflammatory proteins because the knockdown of PGLYRP2/3 are strongly associated with attenuated or increased pro-inflammatory cytokine production. This may account for the observation that why L. acidophilus MYL201 is impotent in development of inflammation tolerance while $L$. plantarum MYL26 is potent because $L$. plantarum MYL26 induces PGLYRP3 while L. acidophilus MYL201 activates NOD2 and PGLYRP2.

Taken together, HepG2 cells up-regulates the expression of PGLYRP3, which is supposed to counteract PGLYRP2 and NOD2, as a self-defence mechanism against $S$. aureus-induced inflammatory process. A rational interpretation for why PGLYRP3 knockdown led to diminished anti-inflammatory effect, while PGLYRP2 knockdown resulted in diminished inflammatory effect, may lie in the fact that PGLYRP3 plays an anti-inflammatory role, whereas PGLYRP2 acts as a pro-inflammatory mediator.

\section{Conclusions}

In conclusion, we discovered that the effects of probiotics in the attenuation of $S$. aureus- induced liver damage are by means of PGLYRP3 activation. The phenomenon of better defence against $S$. aureus challenge in HepG2 cells was attributed to previous induction of PGLYRP3 expression which led to feedback control of NOD2 and PGLYRP2. However, one of the difficulties researchers confront is that the ability for activating the expression of PGLYRP3 varies from strains to strains. Furthermore, inflammation process induction is multi-factorial, and thus there is no single solution to solve such multifaceted problem. This research might give a new insight on screen method establishment based on a novel molecular mechanism for selecting potent probiotic strains with the purpose of conferring hepatoprotective benefit.

\section{References}

Aggarwal, J., Swami, G., \& Kumar, M. (2013). Probiotics and their Effects on Metabolic Diseases: An Update. $\begin{array}{lllll}\text { Journal of clinical and diagnostic research: JCDR, } & 7(1), & 173-177 .\end{array}$ http://dx.doi.org/10.7860/JCDR/2012/5004.2701

Arora, T., Singh, S., \& Sharma, R. K. (2013). Probiotics: Interaction with gut microbiome and antiobesity potential. Nutrition, 29(4), 591-596. http://dx.doi.org/10.1016/j.nut.2012.07.017

Balamayooran, T., Balamayooran, G., \& Jeyaseelan, S. (2010). Review: Toll-like receptors and NOD-like receptors in pulmonary antibacterial immunity. Innateimmunity, 16(3), 201-210. http://dx.doi.org/10.1177/1753425910366058

Blaut, M., \& Klaus, S. (2012). Intestinal microbiota and obesity. Handbook of experimental pharmacology, (209), 251-273. http://dx.doi.org/10.1007/978-3-642-24716-3_11

Boneca, I. G. (2009). Mammalian PGRPs in the spotlight. Cell host \& microbe, 5(2), 109-111. http://dx.doi.org/10.1016/j.chom.2009.01.007 
Brenner, C., Galluzzi, L., Kepp, O., \& Kroemer, G. (2013). Decoding cell death signals in liver inflammation. Journal of hepatology. http://dx.doi.org/10.1016/j.jhep.2013.03.033

Caradonna, L., Amati, L., Magrone, T., Pellegrino, N. M., Jirillo, E., \& Caccavo, D. (2000). Enteric bacteria, lipopolysaccharides and related cytokines in inflammatory bowel disease: biological and clinical significance. Journal of endotoxin research, 6(3), 205-214.

Cartwright, N., Murch, O., McMaster, S. K., Paul-Clark, M. J., van Heel, D. A., Ryffel, B., ... \& Mitchell, J. A. (2007). Selective NOD1 agonists cause shock and organ injury/dysfunction in vivo. American journal of respiratory and critical care medicine, 175(6), 595-603.

Conterno, L., Fava, F., Viola, R., \& Tuohy, K. M. (2011). Obesity and the gut microbiota: does up-regulating colonic fermentation protect against obesity and metabolic disease? Genes \& nutrition, 6(3), 241-260. http://dx.doi.org/10.1007/s12263-011-0230-1

Faria, A. M., Mucida, D., McCafferty, D. M., Tsuji, N. M., \& Verhasselt, V. (2012). Tolerance and inflammation at the gut mucosa. Clinical \& developmental Immunology, 2012, 738475. http://dx.doi.org/10.1155/2012/738475

Foligne, B., Zoumpopoulou, G., Dewulf, J., Ben Younes, A., Chareyre, F., Sirard, J. C., ... Grangette, C. (2007). A key role of dendritic cells in probiotic functionality. PloS one, 2(3), e313.

Franchi, L., Park, J. H., Shaw, M. H., Marina-Garcia, N., Chen, G., Kim, Y. G., \& Nunez, G. (2008). Intracellular NOD-like receptors in innate immunity, infection and disease. Cellular microbiology, 10(1), 1-8.

Fujita, Y., \& Taguchi, H. (2012). Overview and outlook of Toll-like receptor ligand-antigen conjugate vaccines. Therapeutic delivery, 3(6), 749-760.

Inoue, M., \& Shinohara, M. L. (2013). The role of interferon-beta in the treatment of multiple sclerosis and experimental autoimmune encephalomyelitis - in the perspective of inflammasomes. Immunology, 139(1), 11-18. http://dx.doi.org/10.1111/imm.12081

Juarez-Verdayes, M. A., Rodriguez-Martinez, S., Cancino-Diaz, M. E., \& Cancino-Diaz, J. C. (2013). Peptidoglycan and muramyl dipeptide from Staphylococcus aureus induce the expression of VEGF-A in human limbal fibroblasts with the participation of TLR2-NFkappaB and NOD2-EGFR. Graefe's archive for clinical and experimental ophthalmology $=$ Albrecht von Graefes Archiv fur klinische und experimentelle Ophthalmologie, 251(1), 53-62. http://dx.doi.org/10.1007/s00417-012-2130-5

Kim, H. G., Lee, S. Y., Kim, N. R., Lee, H. Y., Ko, M. Y., Jung, B. J., ... Chung, D. K. (2011). Lactobacillus plantarum lipoteichoic acid down-regulated Shigella flexneri peptidoglycan-induced inflammation. Molecular immunology, 48(4), 382-391. http://dx.doi.org/10.1016/j.molimm.2010.07.011

Larsen, N., Vogensen, F. K., van den Berg, F. W., Nielsen, D. S., Andreasen, A. S., Pedersen, B. K., ... Jakobsen, M. (2010). Gut microbiota in human adults with type 2 diabetes differs from non-diabetic adults. PloS one, 5(2), e9085. http://dx.doi.org/10.1371/journal.pone.0009085

Macho Fernandez, E., Valenti, V., Rockel, C., Hermann, C., Pot, B., Boneca, I. G., \& Grangette, C. (2011). Anti-inflammatory capacity of selected lactobacilli in experimental colitis is driven by NOD2-mediated recognition of a specific peptidoglycan-derived muropeptide. Gut, 60(8), 1050-1059. http://dx.doi.org/10.1136/gut.2010.232918

Manco, M., Putignani, L., \& Bottazzo, G. F. (2010). Gut microbiota, lipopolysaccharides, and innate immunity in the pathogenesis of obesity and cardiovascular risk. Endocrine reviews, 31(6), 817-844. http://dx.doi.org/10.1210/er.2009-0030

Matuchansky, C. (2012). Anti-inflammatory lactobacilli: strain specificity. Gut, 61(5), 786; author reply 784. http://dx.doi.org/10.1136/gutjnl-2011-300881

Mitchell, J. A., Paul-Clark, M. J., Clarke, G. W., McMaster, S. K., \& Cartwright, N. (2007). Critical role of toll-like receptors and nucleotide oligomerisation domain in the regulation of health and disease. The Journal of endocrinology, 193(3), 323-330.

Muller-Anstett, M. A., Muller, P., Albrecht, T., Nega, M., Wagener, J., Gao, Q., ... Gotz, F. (2010). Staphylococcal peptidoglycan co-localizes with Nod2 and TLR2 and activates innate immune response via both receptors in primary murine keratinocytes. PloS one, 5(10), e13153. http://dx.doi.org/10.1371/journal.pone.0013153 
Norman, K., \& Pirlich, M. (2008). Gastrointestinal tract in liver disease: which organ is sick? Current opinion in clinical nutrition and metabolic care, 11(5), 613-619. http://dx.doi.org/10.1097/MCO.0b013e32830a70bc

Noval Rivas, M., Burton, O. T., Wise, P., Zhang, Y. Q., Hobson, S. A., Garcia Lloret, M., ... Chatila, T. A. (2013). A microbiota signature associated with experimental food allergy promotes allergic sensitization and anaphylaxis. The Journal of allergy and clinical immunology, 131(1), 201-212. http://dx.doi.org/10.1016/j.jaci.2012.10.026

Quigley, E. M. (2012). Prebiotics and probiotics: their role in the management of gastrointestinal disorders in adults. Nutrition in clinical practice: official publication of the American Society for Parenteral and Enteral Nutrition, 27(2), 195-200. http://dx.doi.org/10.1177/0884533611423926

Scott, M. J., Chen, C., Sun, Q., \& Billiar, T. R. (2010). Hepatocytes express functional NOD1 and NOD2 receptors: a role for NOD1 in hepatocyte CC and CXC chemokine production. Journal of hepatology, 53(4), 693-701. http://dx.doi.org/10.1016/j.jhep.2010.04.026

Seo, Y. S., \& Shah, V. H. (2012). The role of gut-liver axis in the pathogenesis of liver cirrhosis and portal hypertension. Clinical and molecular hepatology, $337-346$. http://dx.doi.org/10.3350/cmh.2012.18.4.337

Shida, K., Kiyoshima-Shibata, J., Kaji, R., Nagaoka, M., \& Nanno, M. (2009). Peptidoglycan from lactobacilli inhibits interleukin-12 production by macrophages induced by Lactobacillus casei through Toll-like receptor 2-dependent and independent mechanisms. Immunology, 128(1 Suppl), e858-869. http://dx.doi.org/10.1111/j.1365-2567.2009.03095.x

Sorbara, M. T., \& Philpott, D. J. (2011). Peptidoglycan: a critical activator of the mammalian immune system during infection and homeostasis. Immunological reviews, 243(1), 40-60. http://dx.doi.org/10.1111/j.1600-065X.2011.01047.x

Stoffels, K., Overbergh, L., Giulietti, A., Kasran, A., Bouillon, R., Gysemans, C., \& Mathieu, C. (2004). NOD macrophages produce high levels of inflammatory cytokines upon encounter of apoptotic or necrotic cells. Journal of autoimmunity, 23(1), 9-15.

Volz, T., Nega, M., Buschmann, J., Kaesler, S., Guenova, E., Peschel, A., ... Biedermann, T. (2010). Natural Staphylococcus aureus-derived peptidoglycan fragments activate NOD2 and act as potent costimulators of the innate immune system exclusively in the presence of TLR signals. FASEB journal: official publication of the Federation of American Societies for Experimental Biology, 24(10), 4089-4102. http://dx.doi.org/10.1096/fj.09-151001

Wang, M., Adawi, D., Molin, G., Pettersson, B., Jeppsson, B., \& Ahrne, S. (2001). Identification of the translocating bacteria in rats with acute liver injury and their relation to the bacterial flora of the intestinal mucosa. APMIS: acta pathologica, microbiologica, et immunologica Scandinavica, 109(7-8), 551-558.

\section{Copyrights}

Copyright for this article is retained by the author(s), with first publication rights granted to the journal.

This is an open-access article distributed under the terms and conditions of the Creative Commons Attribution license (http://creativecommons.org/licenses/by/3.0/). 